\title{
Dimensions of color harmony
}

\author{
DONALD J. POLZELLA and DEMARIS A. MONTGOMERY \\ University of Dayton, Dayton, Ohio
}

\begin{abstract}
Twenty-one subjects rated a random sequence of 144 color pairs on a categorical scale ranging from 1 (harmonizing) to 7 (clashing). Another group of 35 subjects rated each individual color on a set of semantic differential scales. The colors were also scaled objectively in terms of Munsell measures for hue, chroma, and value. The color harmony ratings were analyzed by using multidimensional scaling and invoking a weighted individual-differences Euclidean distance model. A two-dimensional solution accounted for $83.1 \%$ of the variability in the transformed data matrices. On the basis of supplementary regression analyses, it was found that hue, value (brightness), and the psychological attribute "pleasantness," were the factors that influenced the color harmony judgments. In general, harmonious color combinations involved colors that were similar with regard to these attributes.
\end{abstract}

Color harmony, the "suitability" of juxtaposed colors, is a complex phenomenon that is influenced by the individual colors' characteristics (i.e., hue, brightness, saturation) and their relative areas. The most extensive study of this phenomenon was conducted by Helson and Lansford (1970), whose subjects rated the pleasantness of 125 colors on 25 colored backgrounds in five sources of illumination. The principle factor determining the pleasantness of color combinations was brightness contrast-the most pleasant combinations involved large brightness differences between color and background, whereas the least pleasant combinations involved little or no difference.

Hue and saturation contrast were less decisive factors. In the case of saturation, large differences between color and background tended to be judged as being more pleasant than were small differences; however, small differences in saturation could be judged as being pleasant or unpleasant, depending on whether or not there was a high degree of brightness contrast. The findings regarding hue differences were even more equivocal. Earlier studies had shown that the more pleasant combinations involved either zero, small, or large hue differences between color and background, but not intermediate. Helson and Lansford (1970) found that this generalization held for some hue families, but not for others.

Helson and Lansford's (1970) overall conclusion was that color harmony is not easily predicted and depends on the interaction of the spectral characteristics of illumination source, background color, and the hue, brightness, and saturation of the object color. Their conclusion was reinforced in a later study conducted by Pieters (1979), who found that the judgment of color harmony

A preliminary report of this study was presented at the 1989 meeting of the American Psychological Association, New Orleans, LA. D. A. Montgomery is currently a doctoral candidate at the University of Florida in Gainesville. Correspondence concerning this paper should be sent to D. Polzella, Department of Psychology, University of Dayton, 300 College Park, Dayton, OH 45469-1430. was a function of three separate "harmonies," each related to one of the basic parameters. The function, which was derived by using a conjoint measurement algorithm, was expressed as a composition rule consisting of a distributive relationship between saturation harmony and the sum of hue harmony and brightness harmony. This finding implies that the joint effect of hue and brightness harmony changes with variations in saturation harmony.

In addition to the basic color parameters, it is clear that color harmony is also affected by relative area. Morriss and his colleagues demonstrated the importance of "spatial balance" in two experiments (Morriss \& Dunlap, 1987; Morriss, Dunlap, \& Hammond, 1982) in which subjects were able to control the relative areas of juxtaposed colors. They found that saturation and relative area interacted, as well as brightness and relative area. For example, a small area of high saturation (or of high brightness) would appear balanced when juxtaposed with a large area of low saturation (or of low brightness). When colors were closely matched in saturation or in brightness, the subjects tended to choose equal relative areas to achieve balance.

The findings of Morriss (Morriss \& Dunlap, 1987; Morriss, Dunlap, \& Hammond, 1982) suggest that Helson and Lansford's (1970) major conclusion-that pleasant color combinations tend to involve contrasting levels of brightness or saturation-is not generally applicable because it was based on the presentation of relatively small stimuli $(2.4 \times 3.8 \mathrm{~cm}$ color chips $)$ on a relatively large, colored background $(43.2 \times 55.9 \mathrm{~cm})$. The situation is further complicated in that Helson and Lansford simultaneously displayed 12 chips on a background, and their subjects separately rated each chip-background combination.

The present experiment was conducted to help reconcile the findings of both Helson and Lansford (1970) and Morriss (Morriss \& Dunlap, 1987; Morriss, Dunlap, \& Hammond, 1982). Subjects judged the harmony of all possible pairings of 12 equal-sized rectangular color patches varying in hue, saturation, and brightness. In addition, 
they rated the colors singly on 12 semantic differential scales. These ratings, plus Munsell measures of hue, value (brightness), and chroma (saturation), were used to help interpret the harmony judgments.

\section{METHOD}

\section{Subjects}

The subjects were 56 undergraduate student volunteers ( 24 males, 32 females). They were run in two groups of 21 and 35 , respectively.

\section{Stimuli and Apparatus}

The stimuli, $16.5 \times 5.2 \mathrm{~cm}$ rectangular color patches, were displayed against a light, neutral-density background (50\% reflectance) on an IBM Model 5153 color display $(26.7 \times 19.0 \mathrm{~cm})$ driven by an IBM color/ graphics monitor adaptor. The stimuli are listed in Table 1, along with their approximate Munsell equivalents for hue, value (brightness), and chroma (saturation). The right-hand column lists the mean rating of each stimulus on a 7-point pleasant-unpleasant scale. The experiment was controlled by an 8088-based IBM-PC XT computer.

\section{Procedure}

Each subject in the first group $(n=21)$ was shown a random sequence of 144 color pairs, composed of all possible pairings of the 12 stimuli placed one above the other. After the presentation of each pair, the subject was prompted to enter a rating of its suitability on a categorical scale ranging from 1 (harmonizing) to 7 (clashing).

Next, each subject in the second group $(n=35)$ rated the colors singly on 12 semantic differential 7-point scales: blatant-muted, hot-cold, pleasant-unpleasant, hard-soft, active-passive, lush-austere, strongweak, exciting-calming, obvious-subtle, sharp-dull, vibrant-still, and usual-unusual. The scales were those identified by Hogg (1969) as being most applicable to the rating of colors.

\section{RESULTS}

The color harmony ratings were analyzed by means of multidimensional scaling (MDS), a family of statistical techniques that attempts to discover the "hidden structure" in data consisting of measures of relatedness among sets of objects or stimuli (see Schiffman, Reynolds, \& Young, 1981). MDS uses a matrix of proximities among the stimuli as input and produces an $\mathrm{N}$-dimensional configuration or map of the stimuli as output. The configuration is derived in such a way that the distances between the stimuli in the configuration match the original prox-

Table 1

Characteristics of the Color Stimuli

\begin{tabular}{|c|c|c|c|c|c|}
\hline \multirow[b]{2}{*}{ Color } & \multicolumn{4}{|c|}{ Munsell Equivalent* } & \multirow[b]{2}{*}{ Pleasantness $\dagger$} \\
\hline & & Hue & Value & Chroma & \\
\hline Blue & 75 & (5PB) & 4 & 10 & 2.6 \\
\hline Lt. Blue & 75 & (5PB) & 6 & 10 & 2.7 \\
\hline Green & 45 & $(5 G)$ & 6 & 10 & 4.2 \\
\hline Lt. Green & 45 & (5G) & 7 & 10 & 4.0 \\
\hline Cyan & 65 & (5B) & 6 & 6 & 3.6 \\
\hline Lt. Cyan & 65 & (5B) & 7 & 8 & 2.5 \\
\hline Red & 5 & (5R) & 4 & 10 & 4.0 \\
\hline Lt. Red & 5 & (5R) & 5 & 12 & 4.0 \\
\hline Magenta & 85 & (5P) & 4 & 10 & 2.8 \\
\hline Lt. Magenta & 85 & (5P) & 5 & 10 & 2.9 \\
\hline Brown & 25 & (5Y) & 5 & 5 & 5.7 \\
\hline Yellow & 25 & $(5 Y)$ & 8 & 10 & 4.1 \\
\hline
\end{tabular}

*Determined by visual comparison with calibrated color sample chips. † Mean rating on 7-point pleasant-unpleasant scale.

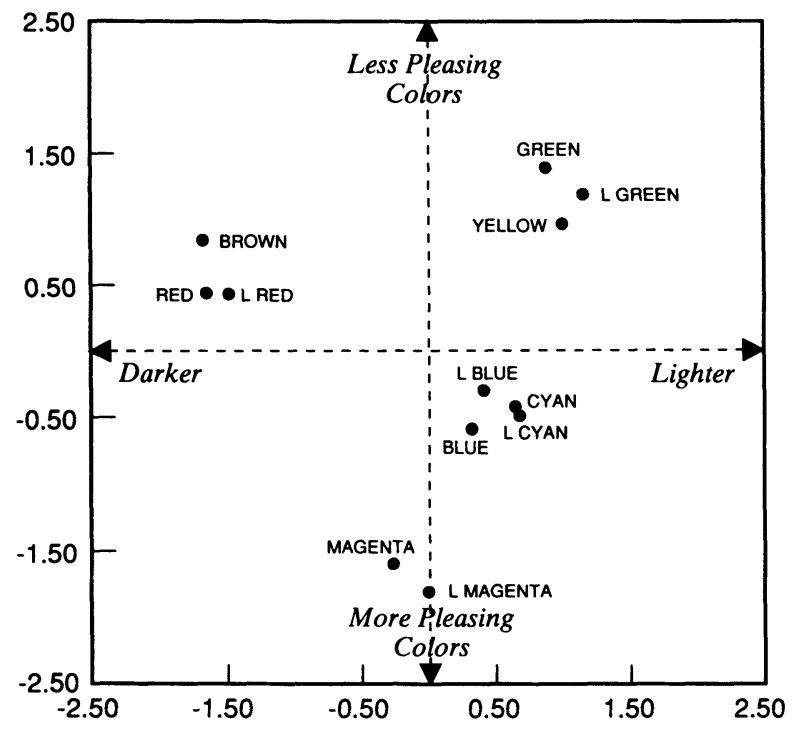

Figure 1. Two-dimensional MDS (INDSCAL) configuration of color harmony judgments.

imity measures as closely as possible. More importantly, the locations of particular clusters of stimuli in the configuration are said to reflect whatever dimensions might underlie the proximity measures.

In the present study, the color harmony data were treated as proximity measures. The (1988) SPSS-X multidimensional scaling procedure ALSCAL was used to analyze these data, which were input as a series of matrices containing each subject's pairwise judgments. A weighted individual-differences Euclidean distance model (INDSCAL) was invoked as the scaling model for the analysis.

A two-dimensional solution accounted for $83.1 \%$ of the variability in the transformed proximity data (averaged over stimuli and matrices). A three-dimensional solution accounted for only $3.7 \%$ additional variability, suggesting that the two-dimensional solution was sufficient and appropriate. The solution is geometrically depicted in Figure 1.

The configuration may be viewed as a color harmony space in which the relative proximities of the individual colors reflect the degree to which they can be combined in a pleasing manner. Thus, juxtaposed blues (i.e., blue and/or cyan) are highly pleasing, whereas a combination of green and magenta is considerably less pleasing.

Statistical interpretation of an MDS configuration can be achieved by several means. The most common approach is to determine whether some objective stimulus parameter(s) can be projected through the space. A technique that is frequently used is to regress a set of candidate stimulus parameters, in turn, on the set of stimulus coordinates. A particular parameter is said to provide a satisfactory interpretation of a dimension if the multiple correlation for the parameter and the regression weight on that dimension are both high (see Kruskal \& Wish, 1978, pp. 36-43, for an example of this approach). In the present study, 
the mean attribute ratings and the Munsell measures comprised the set of candidate stimulus parameters.

The most important factor influencing the color harmony judgments was hue $(R=.92, p<.001)$, which weighted significantly on both dimensions $(p<.005)$. The configuration clearly indicates that, with one exception (i.e., brown and yellow), the most harmonious combinations involved closely related pairs of hues. A second factor influencing the color harmony judgments was value $(R=.80, p<.01)$, which weighted significantly on Dimension 1 (horizontal) $(p<.01)$. High-value colors (i.e., light) tend to be located on the right side of the configuration (or to the right of their identical hue counterpart), whereas low-value colors (i.e., dark) tend to be located on the left side. This indicates that the more harmonious combinations involved colors of similar value.

A final factor that was found to influence the color harmony judgments was the pleasantness of the individual colors $(R=.85, p<.005)$, which weighted significantly on Dimension 2 (vertical) $(p<.005)$. The colors rated as more pleasing tend to be located in the lower half of the configuration, whereas those rated as less pleasing tend to be located in the upper half. This indicates that the more harmonious combinations involved colors that were pleasing to a similar degree, but were not necessarily pleasant individually. Indeed, the configuration reveals that two relatively unpleasant colors, green and yellow, were judged as being harmonious when combined.

\section{DISCUSSION}

The results of this experiment help to clarify and extend what is known about the phenomenon of color harmony. Helson and Lansford's (1970) conclusion-that brightness contrast is the principal factor determining the pleasantness of color combinations - was not supported. Indeed, the evidence presented here suggests that the more harmonious combinations involve colors of similar brightness. It seems plausible that this discrepancy is related to the methodological differences between the two studies. Helson and Lansford's subjects judged the harmony of relatively small colors on a relatively large background, whereas those in the present study judged colors of equal areas.

The present evidence thus supports Morriss and Dunlap (1987), who found that subjects tended to choose equal relative areas when balancing colors that were closely matched in brightness. When colors were of different brightness, subjects tended to avoid equal areas. With regard to brightness, then, it appears that color harmony is achieved when juxtaposed colors are either of similar brightness and similar relative area or of contrasting brightness and contrasting relative area.
The findings regarding the relationship between hue and color harmony are more problematic. Helson and Lansford (1970) noted that earlier studies had shown that pleasant combinations involve zero, small, or large differences in hue, but not intermediate. Helson and Lansford found only minimal support for this generalization. This was also the case in the present study. With one exception (yellow-brown), the most pleasing combinations did involve hues of zero or small differences (e.g., blue-light blue, yellow-green); however, combinations of largely dissimilar hues were generally displeasing (e.g., green-magenta). It is important to note that, due to the relatively small number of stimuli, the present study may not have provided an adequate test of the generalization regarding hue combinations.

The remaining color parameter, saturation, appeared to have no effect on color harmony. This contradicts Morriss et al. (1982), who found that the effects of saturation were analogous to those of brightness. The reason for this discrepancy is unclear but may, perhaps, reflect the particular stimuli used in the present experiment. Table 1 reveals that two thirds of the stimuli are of equivalent chroma (saturation). Given the lack of variability, it is hardly surprising that no effects were found.

An unexpected and intriguing finding was that harmonious color combinations involved colors that were similar in pleasantness. The reason for this finding is uncertain, but it may be related to the Gestalt principle of similarity, which is thought to be a fundamental unifying perceptual force (see Arnheim, 1974, pp. 79-92). The principle may help to explain the other findings as well; harmonious color combinations involved colors that were similar in brightness or similar in hue.

\section{REFERENCES}

Arnheim, R. (1974). Art and visual perception (rev. ed.). Berkeley: University of California Press.

HeLson, H., \& LANSFORD, T. (1970). The role of spectral energy of source and background color in the pleasantness of object colors. Applied Optics, 9, 1513-1539.

HoGG, J. (1969). A principal components analysis of semantic differential judgments of single colors and color pairs. Journal of General Psychology, 80, 129-140.

KruSKAL, J., \& WISH, M. (1978). Multidimensional scaling. Beverly Hills, CA: Sage Publications.

Morriss, R. H., \& DUNLAP, W. P. (1987). Influence of value on spatial balance of color pairs. Journal of General Psychology, 114, 353-361.

Morriss, R. H., DunlaP, W. P., \& Hammond, E. H. (1982). Influence of chroma on spatial balance of complementary hues. American Journal of Psychology, 95, 323-332.

Pieters, J. M. (1979). A conjoint measurement approach to color harmony. Perception \& Psychophysics, 26, 281-286.

Schiffman, S. S., Reynolds, M. L., \& Young, F. W. (1981). Introduction to multidimensional scaling: Theory, methods, and applications. New York: Academic Press.

SPSS INC. (1988). SPSS-X user's guide (3rd ed.). Chicago, IL: Author.

(Manuscript received April 26, 1993.) 